\title{
RACSAMI
}

Rev. R. Acad. Cien. Serie A. Mat.

VOL. 103 (2), 2009, pp. 219-234

Matemática Aplicada / Applied Mathematics

\section{Duality results involving functions associated to nonempty subsets of locally convex spaces}

\author{
C. Zălinescu
}

\begin{abstract}
In many papers on consumer theory and production analysis duality results between profit, revenue, cost, input, output and shortage functions are established. This functions are associated to certain subsets of $\mathbb{R}^{n}$. The aim of this paper is to study in a systematic way such duality results in locally convex spaces and to derive them under minimal hypotheses.
\end{abstract}

\section{Resultados sobre dualidad mediante funciones asociadas a subconjuntos no vacios de espacios localmente convexos}

Resumen. En muchos artículos sobre teoría del consumo y análisis de la producción, se establecen resultados de dualidad entre beneficios y costes, e inversiones y rendimientos, proponiéndose diversas funciones de insuficiencia asociadas a ciertos subconjuntos de $\mathbb{R}^{n}$. El objeto de este trabajo es el estudio sistemático de dichos resultados de dualidad en espacios localmente convexos, y su obtención bajo condiciones mínimas.

\section{Introduction}

In the sequel $(X, \tau)$ is a nontrivial separated real locally convex space with topological dual $X^{*} ; X^{*}$ is endowed with the weak-star topology $w^{*}:=\sigma\left(X^{*}, X\right)$. So $X^{*}$ becomes a separated locally convex space whose topological dual is (identified with) $X$. For $x \in X$ and $x^{*} \in X^{*}$ we set $\left\langle x, x^{*}\right\rangle:=x^{*}(x)$. In the case $X$ is just a real linear space we can see $X$ as a separated locally convex space whose topology is generated by the family of all semi-norms defined on $X$; in this situation the topological dual of $X$ coincides with the algebraic dual $X^{\prime}$ of $X$. We denote by $\mathbb{R}$ the set of real numbers, $\mathbb{R}_{+}:=\left[0, \infty\left[, \mathbb{R}_{-}:=\right]-\infty, 0\right]$ and $\mathbb{P}:=] 0, \infty[$, where $\infty:=+\infty$.

Consider $A, B \subset X$ and $\Gamma \subset \mathbb{R}$ (similar for $X$ replaced by $X^{*}$ or other locally convex space). We set

$$
A+B:=\{a+b \mid a \in A, b \in B\}, \quad \Gamma A:=\{s a \mid s \in \Gamma, a \in A\} ;
$$

of course, $A+B=\emptyset$ if $A=\emptyset$ or $B=\emptyset$ and $\Gamma A=\emptyset$ when $\Gamma=\emptyset$ or $A=\emptyset$. Moreover, for $s \in \mathbb{R}$ we set $s A:=\{s\} A$ and for $x \in X$ we set $x+A:=\{x\}+A$. We denote by ${ }^{i} A$ or icr $A$, int $A, \operatorname{cl} A$ or $\bar{A}$, conv $A$ and aff $A$ the intrinsic core, the interior, the closure, the convex hull and the affine hull of $A \subset X$, respectively; moreover, $\overline{\operatorname{conv}} A:=\operatorname{cl}(\operatorname{conv} A)$ and $\overline{\operatorname{aff}} A:=\operatorname{cl}(\operatorname{aff} A)$. To $A \subset X$ we associate the sets

$$
\begin{aligned}
& A^{0}:=\left\{x^{*} \in X^{*} \mid\left\langle x, x^{*}\right\rangle \geq-1 \forall x \in A\right\}, \\
& A^{+}:=\left\{x^{*} \in X^{*} \mid\left\langle x, x^{*}\right\rangle \geq 0 \forall x \in A\right\}, \quad A^{-}:=-A^{+}, \\
& A^{\#}:=\left\{x^{*} \in X^{*} \mid\left\langle x, x^{*}\right\rangle>0 \forall x \in A \backslash\{0\}\right\} .
\end{aligned}
$$

Presentado por / Submitted by Pedro Jiménez Guerra.

Recibido /Received: May 16, 2008. Aceptado /Accepted: March 4, 2009.

Palabras clave / Keywords: Duality results, localy convex spaces, shortage functions, profit, revenue, input, output.

Mathematics Subject Classifications: 49N15, 90C46, 91B38.

(C) 2009 Real Academia de Ciencias, España. 
Note that $A^{0}$ is a $\left(w^{*}\right)$ closed convex set containing $0, A^{+}$is a $\left(w^{*}-\right)$ closed convex cone and $A^{\#}$ is a convex cone (if nonempty). For $\emptyset \neq A \subset X$ the bipolar theorems give

$$
A^{00}=\overline{\operatorname{conv}}(A \cup\{0\})=\overline{\operatorname{conv}}([0,1] A), \quad A^{++}=A^{--}=\overline{\operatorname{conv}}\left(\mathbb{R}_{+} A\right) .
$$

These formulas give the possibility to recover $A$ using the above polarity operations under certain conditions on $A$ : $A=A^{00}$ if (and only if) $A$ is a closed convex set containing 0 and $A=A^{++}$if (and only if) $A$ is a closed convex cone.

The asymptotic cone of the nonempty set $A \subset X$ is

$$
A_{\infty}:=\left\{u \in X \mid \exists\left(t_{i}\right)_{i \in I} \subset \mathbb{P}, \exists\left(x_{i}\right)_{i \in I} \subset A: t_{i} \rightarrow 0, t_{i} x_{i} \rightarrow u\right\}
$$

when $X$ is a normed vector space we can take sequences instead of nets. When $A$ is a closed convex set we have that $A_{\infty}=\bigcap_{t>0} t(A-a)$, where $a \in A$. We set $\emptyset_{\infty}:=\{0\}$.

Recall that for $f: X \rightarrow \overline{\mathbb{R}}:=\mathbb{R} \cup\{-\infty, \infty\}$ the domain of $f$ is the set $\operatorname{dom} f:=\{x \in X \mid$ $f(x)<\infty\}$ and the epigraph of $f$ is the set epi $f:=\{(x, t) \in X \times \mathbb{R} \mid f(x) \leq t\} ; f$ is proper if $\operatorname{dom} f \neq \emptyset$ and $f(x)>-\infty$ for every $x \in X$. The conjugate $f^{*}: X^{*} \rightarrow \overline{\mathbb{R}}$ of $f$ is defined by $f^{*}\left(x^{*}\right):=\sup \left\{\left\langle x, x^{*}\right\rangle-f(x) \mid x \in X\right\} ; f^{*}$ is convex and weakly-star lower semicontinuous (lsc for short) and $f^{*}$ is proper iff $f$ is proper and minorized by a continuous affine functional (in which case epi $f^{* *}=\overline{\operatorname{conv}}($ epi $f)$ ). We denote by $\bar{f}$ the function $\bar{f}: X \rightarrow \overline{\mathbb{R}}$ for which epi $\bar{f}:=\operatorname{cl}($ epi $f)$; then $\bar{f}(x)=\liminf _{x^{\prime} \rightarrow x} f\left(x^{\prime}\right)$. Furthermore, we use the notation $[f \leq t]:=\{x \in X \mid f(x) \leq t\}$ for $t \in \mathbb{R}$, and similarly for $[f=t],[f \geq t],[f<t],[f>t]$. If $g: X \rightarrow \overline{\mathbb{R}}$ is another function then the convolution of $f$ and $g$ is the function $f \square g: X \rightarrow \overline{\mathbb{R}}$ defined by $(f \square g)(x):=\inf \left\{f(u)+_{e} g(x-u) \mid u \in X\right\}$ (the sum " $+e$ " is defined in the next section).

\section{Gauges and scalarization functions}

Let $A \subset X$ be an arbitrary set. First we associate to $A$ the following two functions

$$
\sigma_{A}, \varsigma_{A}: X^{*} \rightarrow \overline{\mathbb{R}}, \quad \sigma_{A}\left(x^{*}\right):=\sup _{x \in A}\left\langle x, x^{*}\right\rangle, \quad \varsigma_{A}\left(x^{*}\right):=\inf _{x \in A}\left\langle x, x^{*}\right\rangle
$$

with the conventions $\sup \emptyset:=-\infty$ and $\inf \emptyset:=\infty$; hence $\sigma_{\emptyset}=-\infty$ and $\varsigma \emptyset=+\infty$. The function $\sigma_{A}$ is the support function of $A$. It is obvious that

$$
\varsigma_{A}\left(x^{*}\right)=-\sigma_{A}\left(-x^{*}\right) \quad \forall x^{*} \in X^{*}
$$

moreover $\sigma_{A}=\sigma_{\overline{\operatorname{conv}} A}, \varsigma_{A}=\varsigma_{\overline{\operatorname{conv}} A}$ and

$$
\begin{aligned}
& \overline{\operatorname{conv}} A=\left\{x \in X \mid\left\langle x, x^{*}\right\rangle \leq \sigma_{A}\left(x^{*}\right) \quad \forall x^{*} \in X^{*}\right\} \\
& =\left\{x \in X \mid\left\langle x, x^{*}\right\rangle \geq \varsigma_{A}\left(x^{*}\right) \quad \forall x^{*} \in X^{*}\right\} .
\end{aligned}
$$

The above formulas show that we can recover $A$ knowing $\sigma_{A}$ or $\varsigma_{A}$ when $A$ is a (nonempty) closed convex set.

Denoting by $\iota_{A}$ the indicator function of $A \subset X$, that is, $\iota_{A}: X \rightarrow \overline{\mathbb{R}}$ is defined by $\iota_{A}(x):=0$ for $x \in A$ and $\iota_{A}(x):=\infty$ for $x \in X \backslash A$, it is clear that $\sigma_{A}:=\iota_{A}^{*}:=\left(\iota_{A}\right)^{*}$. When $A$ is a nonempty set $\sigma_{A}$ is a proper $w^{*}$-lsc sublinear functional.

To $A \subset X$ we associate also the gauges $\mu_{A}, \vartheta_{A}, \nu_{A}, \theta_{A}: X \rightarrow \overline{\mathbb{R}}$ defined by

$$
\begin{array}{ll}
\mu_{A}(x):=\inf \{\lambda>0 \mid x \in \lambda A\}, & \vartheta_{A}(x):=\sup \{\lambda>0 \mid \lambda x \in A\}, \\
\nu_{A}(x):=\sup \{\lambda>0 \mid x \in \lambda A\}, & \theta_{A}(x):=\inf \{\lambda>0 \mid \lambda x \in A\} ;
\end{array}
$$


hence $\mu_{\emptyset}=\theta_{\emptyset}=+\infty$ and $\nu_{\emptyset}=\vartheta_{\emptyset}=-\infty$. Note that $\mu_{A}$ is the well known Minkowski functional associated to $A$; in Functional Analysis $\mu_{A}$ is considered mostly when $A$ is an absorbing convex set in which case $\mu_{A}$ is a finite-valued sublinear function. Shephard's input and output functions are of type $\nu_{A}$ and $\mu_{A}$ with $A$ subsets of $\mathbb{R}_{+}^{n}$. For some properties of $\mu_{A}$ and $\nu_{A}$ see f.i. [12]. In [5] one speaks about $\theta_{A}$ as the extended Farrell measure; moreover, in [5] one discusses arguments in favor of and against convexity axioms in DEA (Data Envelopment Analysis).

Note that the functions $\theta_{A}, \mu_{A}$ are finite at $x \in X \backslash\{0\}$ if and only if $x \in \mathbb{P} A$. Moreover, $\mu_{A}=$ $\mu_{] 0,1] A} \geq 0, \vartheta_{A}=\vartheta_{] 0,1] A}, \nu_{A}=\nu_{[1, \infty[A}, \theta_{A}=\theta_{[1, \infty[A} \geq 0$,

$$
\begin{array}{lll}
\mu_{A}(t x)=t \mu_{A}(x), & \nu_{A}(t x)=t \nu_{A}(x) & \forall x \in X, \forall t \in \mathbb{P}, \\
\theta_{A}(t x)=t^{-1} \theta_{A}(x), & \vartheta_{A}(t x)=t^{-1} \vartheta_{A}(x) & \forall x \in X, \forall t \in \mathbb{P}
\end{array}
$$

and

$$
\mu_{A}(x) \leq \nu_{A}(x), \quad \theta_{A}(x)=1 / \nu_{A}(x), \quad \vartheta_{A}(x)=1 / \mu_{A}(x) \quad \forall x \in \mathbb{P} A
$$

with the conventions $1 / \infty:=0$ and $1 / 0:=\infty$. In fact

$$
\mu_{A}(x)=\frac{1}{0 \vee \vartheta_{A}(x)}, \quad 0 \vee \vartheta_{A}(x)=\frac{1}{\mu_{A}(x)}, \quad \theta_{A}(x)=\frac{1}{0 \vee \nu_{A}(x)}, \quad 0 \vee \nu_{A}(x)=\frac{1}{\theta_{A}(x)}
$$

for every $x \in X$, where $s \vee t:=\max \{s, t\}$ for $s, t \in \overline{\mathbb{R}}$. We use also the conventions

$$
\begin{array}{ll}
0 \cdot \cdot_{e} \infty:=\infty \cdot{ }_{e} 0:=\infty, & 0 \cdot_{e}(-\infty):=(-\infty) \cdot \cdot_{e} 0:=0, \\
0 \cdot{ }_{h} \infty:=\infty \cdot{ }_{h} 0:=0, & 0 \cdot{ }_{h}(-\infty):=(-\infty) \cdot{ }_{h} 0:=-\infty
\end{array}
$$

(the indexes $e$ and $h$ are coming from epigraph and hypograph, respectively). We also use the conventions

$$
(-\infty)+{ }_{e}(+\infty):=(+\infty)+{ }_{e}(-\infty):=+\infty, \quad(-\infty)+{ }_{h}(+\infty):=(+\infty)+{ }_{h}(-\infty):=-\infty
$$

$(s+t$ being defined as usual in the other situations).

As for other operations on sets, one may ask when and how we can recover the set $A$ knowing $\mu_{A}, \nu_{A}$, $\theta_{A}$ or $\vartheta_{A}$. We have

$$
\begin{array}{ll}
A=\left\{x \in X \mid \mu_{A}(x) \leq 1\right\}=\left\{x \in X \mid \vartheta_{A}(x) \geq 1\right\} & \text { if } A=\operatorname{cl} A=[0,1] A \\
A=\left\{x \in X \mid \nu_{A}(x) \geq 1\right\}=\left\{x \in X \mid \theta_{A}(x) \leq 1\right\} & \text { if } A=\operatorname{cl} A=[1, \infty[A .
\end{array}
$$

In fact, instead of asking $A=\operatorname{cl} A$ we can assume that $A$ is radially closed, or more precisely, that $A \cap \mathbb{R} x$ is closed for every $x \in X$; moreover, in such a case, the non zero finite values of these functions are attained.

Having now the set $A \subset X$ and the element $k \in X \backslash\{0\}$ we consider the function

$$
\varphi_{A, k}: X \rightarrow \overline{\mathbb{R}}, \quad \varphi_{A, k}(x):=\inf \{t \in \mathbb{R} \mid x \in t k-A\}
$$

and its counterpart

$$
\psi_{A, k}: X \rightarrow \overline{\mathbb{R}}, \quad \psi_{A, k}(x):=\sup \{t \in \mathbb{R} \mid x \in t k+A\}
$$

hence $\varphi_{\emptyset, k}=+\infty$ and $\psi_{\emptyset, k}=-\infty$. Note that $\varphi_{A, k}(x)>-\infty$ and $\psi_{A, k}(x)<\infty$ for every $x \in X$ if $k \notin-A_{\infty}$. Moreover,

$$
\begin{gathered}
\varphi_{A, s k}=s^{-1} \varphi_{A, k}, \quad \psi_{A, s k}=s^{-1} \psi_{A, k} \quad \forall s \in \mathbb{P}, \\
A \subset B \Rightarrow\left[\varphi_{B, k} \leq \varphi_{A, k}, \quad \psi_{A, k} \leq \psi_{B, k}\right], \\
\psi_{A, k}=\psi_{A+\mathbb{R}_{+}, k}, \quad \varphi_{A, k}=\varphi_{A+\mathbb{R}_{+} k, k}, \\
A+\mathbb{R}_{+} k \subset\left[\psi_{A, k} \geq 0\right], \quad-A-\mathbb{R}_{+} k \subset\left[\varphi_{A, k} \leq 0\right]
\end{gathered}
$$


and

$$
\psi_{A, k}(x)=-\varphi_{-A,-k}(x)=-\varphi_{A, k}(-x) \quad \forall x \in X .
$$

For this reason it is sufficient to study $\varphi_{A, k}$ or $\psi_{A, k}$. A detailed study of the function $\varphi_{A, k}$ in the case $A$ closed and $A=A+\mathbb{R}_{+} k$ is performed in [9, Section 2.3]; other properties of $\varphi_{A, k}$ are established in [13].

It is known and easy to prove that

$$
\varphi_{A, k}(x+t k)=\varphi_{A, k}(x)+t, \quad \psi_{A, k}(x+t k)=\psi_{A, k}(x)+t \quad \forall x \in X, \quad \forall t \in \mathbb{R} .
$$

Of course, if $A$ is closed (or more generally, $A$ is closed in the direction $k$, that is, $\{t \in \mathbb{R} \mid x+t k \in A\}$ is closed in $\mathbb{R}$ for every $x \in X)$ and $\varphi_{A, k}(x) \in \mathbb{R}$ then $x \in \varphi_{A, k}(x) k-A$, that is, the finite values of $\varphi_{A, k}$ are attained. Moreover, if $A+\mathbb{R}_{+} k$ is closed then

$$
A+\mathbb{R}_{+} k=\left\{x \in X \mid \varphi_{A, k}(-x) \leq 0\right\}=\left\{x \in X \mid \psi_{A, k}(x) \geq 0\right\} .
$$

The function $\varphi_{A, k}$ was introduced by Gerstewitz (Tammer) and Iwanow in [8] and used by Chr. Tammer and her collaborators, as well as by D. T. Luc and others, mainly for scalarization of vector optimization problems; the framework was that of an ordered topological vector space. Luenberger [11, Def. 4.1] considered (practically) the same function, under the name of shortage function, in the context of production analysis ( $X$ being $\mathbb{R}^{n}$ and $A$ a convex subset of $\mathbb{R}_{+}^{n}$ ) and Artzner et. al. [2] considered it in the context of mathematical finance ( $X$ being a space of Lebesgue integrable functions and $A$ the corresponding positive cone). More historical facts about the use of the functions $\varphi_{A, k}, \psi_{A, k}$ in Functional Analysis and Mathematical Economics are given by A. H. Hamel in [10].

In production analysis the condition $A=A+\mathbb{R}_{+} k$ is not granted. Because sometimes the results are not established in very precise terms in this context, the next three statements refer to the case when $A$ might be different of $A+\mathbb{R}_{+} k$. In the sequel we shall omit $k$ if confusions cannot arrive (mainly in the proofs), that is, we shall write simply $\varphi_{A}$ instead of $\varphi_{A, k}$ and $\psi_{A}$ instead of $\psi_{A, k}$.

Proposition 1 Assume that $A \subset X$ is closed, $K \subset X$ and $k \in X \backslash\{0\}$. If

$$
A=\left(A+\mathbb{R}_{+} k\right) \cap K,
$$

then

$$
A=\left\{x \in K \mid \psi_{A, k}(x) \geq 0\right\}=\left\{x \in K \mid \varphi_{A, k}(-x) \leq 0\right\} .
$$

Proof. If $A=\emptyset$ then clearly (11) holds. Assume that $A \neq \emptyset$. The inclusion $A \subset\left\{x \in K \mid \psi_{A}(x) \geq 0\right\}$ is clear. Take $x \in K$ with $\psi_{A}(x) \geq 0$. If $\psi_{A}(x)=0$, since $A$ is closed, we have that $x=x+\psi_{A}(x) k \in A$. Otherwise there exists $t>0$ such that $x-t k \in A$. Then $x=x-t k+t k \in A+\mathbb{R}_{+} k$, and so, by (10), $x \in A$.

When $K$ is a cone and $k \in K$ condition (10) is implied by the condition $(A+K) \cap K=A$, while in the case $-k \in K$ condition (10) is implied by $(A-K) \cap K=A$. Note that in [1] one uses sets $L_{i} \subset \mathbb{R}^{r}$ and $P_{i} \subset \mathbb{R}^{s}$ with the properties $\left(L_{i}+\mathbb{R}_{+}^{r}\right) \cap \mathbb{R}_{+}^{r}=L_{i}$-called free disposability for inputsand $\left(P_{i}-\mathbb{R}_{+}^{s}\right) \cap \mathbb{R}_{+}^{s}=P_{i}$ —called free disposability for outputs.

As seen in (8), the set $\left[\psi_{A} \geq 0\right]$ includes always the set $A+\mathbb{R}_{+} k$, and so it might be different of $A$ even if $A$ is closed and satisfies (10). Take $K:=\mathbb{R}_{+}^{2} \subset X:=\mathbb{R}^{2}, k:=(-1,-1), A:=[0,1] \times[0,1]$; then $\left[\psi_{A} \geq 0\right]=A+\mathbb{R}_{+} k \neq A$. It is clear that the hypotheses of Proposition 1 are satisfied. Relation (2) in [7] could give the impression that $A=\left[\psi_{A} \geq 0\right]$ for $A$ a closed convex set without asking $A=A+\mathbb{R}_{+} k$. As observed in (6) we have that $A \subset B \subset X$ implies $\psi_{A} \leq \psi_{B}$. Applying the previous proposition with $K=X$, if $A$ and $B$ are closed sets (in the direction $k$ ) such that $A=A+\mathbb{R}_{+} k$ and $B=B+\mathbb{R}_{+} k$, then $A \subset B$ if and only if $\psi_{A} \leq \psi_{B}$.

Proposition 2 Assume that $k \in X \backslash\{0\}$ and $A_{i} \subset X$ satisfies (10) for every $i \in I(\neq \emptyset)$. Then $A:=\bigcap_{i \in I} A_{i}$ and $A^{\prime}:=\bigcup_{i \in I} A_{i}$ satisfy (10), too. 
PRoof. Since $A_{i} \subset K$ for every $i \in I$, we have that $A, A^{\prime} \subset K$. Hence $A \subset\left(A+\mathbb{R}_{+} k\right) \cap K$ and $A^{\prime} \subset\left(A^{\prime}+\mathbb{R}_{+} k\right) \cap K$. Obviously, $\left(A+\mathbb{R}_{+} k\right) \cap K \subset\left(A_{i}+\mathbb{R}_{+} k\right) \cap K=A_{i}$ for every $i \in I$, and so $\left(A+\mathbb{R}_{+} k\right) \cap K \subset A$. Let $x \in\left(A^{\prime}+\mathbb{R}_{+} k\right) \cap K$. Then $x=a^{\prime}+t k \in K$ for some $a^{\prime} \in A^{\prime}$ and $t \geq 0$. Hence there exists $i \in I$ with $a^{\prime} \in A_{i}$, and so $x \in K \cap\left(A_{i}+\mathbb{R}_{+} k\right)=A_{i} \subset A^{\prime}$. The conclusion follows.

Condition (10) will be used later on, too.

Proposition 3 Let I be a nonempty set and $A_{i} \subset X$ for every $i \in I$. Then

$$
\psi_{\cup_{i \in I} A_{i}, k}=\sup _{i \in I} \psi_{A_{i}, k}, \quad \varphi_{\cup_{i \in I} A_{i}, k}=\inf _{i \in I} \varphi_{A_{i}, k}, \quad \psi_{\bigcap_{i \in I} A_{i}, k} \leq \inf _{i \in I} \psi_{A_{i}, k}, \quad \varphi_{\bigcap_{i \in I} A_{i}, k} \geq \sup _{i \in I} \varphi_{A_{i}, k} .
$$

Moreover, if $K \subset X$ is closed and $\left(A_{i}+\mathbb{R}_{+} k\right) \cap K=A_{i}$ (that is, $A_{i}$ verifies (10)) for every $i \in I$, then

$$
\psi_{\cap_{i \in I} A_{i}, k}=\inf _{i \in I} \psi_{A_{i}, k}, \quad \varphi_{\cap_{i \in I} A_{i}, k}=\sup _{i \in I} \varphi_{A_{i}, k} .
$$

In particular, if $A_{i}=A_{i}+\mathbb{R}_{+} k$ for every $i \in I$ then (12) holds.

Proof. Because $A:=\bigcap_{i \in I} A_{i} \subset A_{j} \subset \bigcup_{i \in I} A_{i}=: A^{\prime}$ for $j \in I$ we have that $\psi_{A} \leq \psi_{A_{j}} \leq \psi_{A^{\prime}}$ for $j \in I$. This implies that $\psi_{A} \leq \inf _{i \in I} \psi_{A_{i}} \leq \sup _{i \in I} \psi_{A_{i}} \leq \psi_{A^{\prime}}$. On the other hand it is clear that

$$
\left\{t \in \mathbb{R} \mid x-t k \in \bigcup_{i \in I} A_{i}\right\}=\bigcup_{i \in I}\left\{t \in \mathbb{R} \mid x-t k \in A_{i}\right\},
$$

whence $\psi_{A^{\prime}}=\sup _{i \in I} \psi_{A_{i}}$. Similarly, $\varphi_{A^{\prime}}=\inf _{i \in I} \varphi_{A_{i}}$.

Assume now that $K$ is closed and $\left(A_{i}+\mathbb{R}_{+} k\right) \cap K=A_{i}$ for every $i \in I$. Consider $x \in X$ and $s \in \mathbb{R}$ such that $s<\inf _{i \in I} \psi_{A_{i}}(x)$. Hence, for every $i \in I$ we have $s<\psi_{A_{i}}(x)$, and so there exists $t_{i}>s$ such that $x-t_{i} k \in A_{i} \subset K$. Set $\bar{s}:=\inf \left\{t_{i} \mid i \in I\right\} \geq s$. Hence $x-\bar{s} k=x-t_{i} k+\left(t_{i}-\bar{s}\right) k \in A_{i}+\mathbb{R}_{+} k$ for every $i \in I$. Moreover, we have that $x-\bar{s} k \in \operatorname{cl} K=K$. Hence $x-\bar{s} k \in\left(A_{i}+\mathbb{R}_{+} k\right) \cap K=A_{i}$ for every $i \in I$, and so $x-\bar{s} k \in A$. This shows that $\psi_{A}(x) \geq \bar{s} \geq s$. Hence $\psi_{A}=\inf _{i \in I} \psi_{A_{i}, k}$.

A similar argument yields $\varphi_{A}=\sup _{i \in I} \varphi_{A_{i}, k}$.

\section{Duality relations involving the functions $\sigma_{A}, \varsigma_{A}, \theta_{A}$ and $\vartheta_{A}$}

First we establish formulas for $\varsigma_{A}\left(x^{*}\right)$ with $x^{*} \in A^{+}$.

Proposition 4 Let $A \subset X$ and set $\widetilde{A}:=\overline{\operatorname{conv}}\left(\left[1, \infty[A)\right.\right.$. Then for every $x^{*} \in A^{+}$one has

$$
\begin{aligned}
\varsigma_{A}\left(x^{*}\right) & =\inf \left\{\theta_{A}(x) \cdot\left\langle x, x^{*}\right\rangle \mid x \in \mathbb{P} A\right\} \\
& =\inf \left\{\theta_{A}(x) \cdot\left\langle x, x^{*}\right\rangle \mid x \in A\right\} \\
& =\inf \left\{\theta_{A}(x) \cdot{ }_{e}\left\langle x, x^{*}\right\rangle \mid x \in A^{++}\right\} \\
& =\inf \left\{\theta_{\widetilde{A}}(x) \cdot{ }_{e}\left\langle x, x^{*}\right\rangle \mid x \in A^{++}\right\} .
\end{aligned}
$$

Proof. If $A=\emptyset$ (13) holds taking into account our conventions. Assume that $A \neq \emptyset$. Let $x^{*} \in A^{+}$. Then

$$
\begin{aligned}
\varsigma_{A}\left(x^{*}\right) & =\inf \left\{\left\langle x^{\prime}, x^{*}\right\rangle \mid x^{\prime} \in A\right\} \\
& =\inf \left\{t\left\langle x, x^{*}\right\rangle \mid t>0, x \in X, t x=x^{\prime} \in A\right\} \\
& =\inf \left\{t\left\langle x, x^{*}\right\rangle \mid t>0, x \in \mathbb{P} A, t x \in A\right\} \\
& =\inf _{x \in \mathbb{P} A}\left[\left\langle x, x^{*}\right\rangle \cdot \inf \{t>0 \mid t x \in A\}\right] \\
& =\inf \left\{\theta_{A}(x) \cdot\left\langle x, x^{*}\right\rangle \mid x \in \mathbb{P} A\right\} .
\end{aligned}
$$


For the second equality one uses (2), while the third is a rewriting of the first one because for $x \in$ $A^{++} \backslash \mathbb{P} A$ we have that $\theta_{A}(x)=\infty$ and $\left\langle x, x^{*}\right\rangle \geq 0$. It is clear that $A^{+}=\widetilde{A}^{+}$and $\varsigma_{A}\left(x^{*}\right)=\varsigma_{\widetilde{A}}\left(x^{*}\right)$ for $x^{*} \in A^{+}$. Hence the last equality of (13) follows from the previous one replacing $A$ by $\widetilde{A}$.

The next result establishes an estimate for $\varsigma_{A}\left(x^{*}\right)$ when $x^{*} \in X^{*}$.

Proposition 5 Let $\emptyset \neq A \subset X$. Then

$$
\varsigma_{A}\left(x^{*}\right) \leq \inf \left\{\theta_{A}(x) \mid x \in \mathbb{P} A,\left\langle x, x^{*}\right\rangle=1\right\}
$$

for every $x^{*} \in X^{*}$, the inequality being strict for $x^{*} \in\left(X^{*} \backslash A^{+}\right) \cup\left(A^{+} \cap A^{-}\right)$. Moreover, if $0 \notin A$ then

$$
\varsigma_{A}\left(x^{*}\right)=\inf \left\{\theta_{A}(x) \mid x \in \mathbb{P} A,\left\langle x, x^{*}\right\rangle=1\right\}=\inf \left\{\theta_{A}(x) \mid x \in\left[x^{*}=1\right]\right\}
$$

for every $x^{*} \in A^{\#}$.

Proof. For $x^{*} \in A^{+}$(14) follows immediately from (13). If $x^{*} \notin A^{+}$then $\varsigma_{A}\left(x^{*}\right)<0 \leq \lambda\left(x^{*}\right):=$ $\inf \left\{\theta_{A}(x) \mid x \in \mathbb{P} A,\left\langle x, x^{*}\right\rangle=1\right\}$; if $x^{*} \in A^{+} \cap A^{-}$then $\varsigma_{A}\left(x^{*}\right)=0<\infty=\lambda\left(x^{*}\right)$. Assume that $0 \notin A$ and fix $x^{*} \in A^{\#}$. Since $\left\langle x, x^{*}\right\rangle>0$ for every $x \in \mathbb{P} A$, using (2) we may take $\left\langle x, x^{*}\right\rangle=1$ in the second term of (13), getting so (15).

Assuming that $0 \notin A$, the natural question is if (15) is true for $x^{*} \in A^{+} \backslash A^{-}\left(\supset A^{\#}\right)$. We give an affirmative answer under some additional conditions.

Proposition 6 Let $A \subset X \backslash\{0\}$ be a convex set with ${ }^{i} A \neq \emptyset$. Then

$$
\varsigma_{A}\left(x^{*}\right)=\inf \left\{\theta_{A}(x) \mid x \in \mathbb{P} A,\left\langle x, x^{*}\right\rangle=1\right\}=\inf \left\{\theta_{A}(x) \mid x \in\left[x^{*}=1\right]\right\} \quad \forall x^{*} \in A^{+} \backslash A^{-} .
$$

Proof. Set $A_{0}:={ }^{i} A$. Then $A_{0}^{+}=A^{+}, \varsigma_{A_{0}}=\varsigma_{A}$ and $\theta_{A_{0}}(x)=\theta_{A}(x)$ for every $x \in \mathbb{P} A_{0}$. Moreover, $A^{+} \backslash A^{-} \subset A_{0}^{\#}$. The first two assertions follow from the fact that $(1-t) x+t y \in{ }^{i} A$ for $x \in{ }^{i} A, y \in A$ and $t \in\left[0,1\left[\right.\right.$. Since $A_{0} \subset A$ it is clear that $\theta_{A_{0}} \geq \theta_{A}$. Take $x=s \bar{x}$ with $s \in \mathbb{P}$ and $\bar{x} \in A_{0}$, and $\theta_{A}(x)<\bar{t}$. Then there exists $t \in] 0, \bar{t}\left[\right.$ with $t x \in A$. Then $r t x+(1-r) \bar{x}=\left(r t+(1-r) s^{-1}\right) x \in A_{0}$ for all $\left.r \in\right] 0,1[$. Hence $\theta_{A_{0}}(x) \leq r t+(1-r) s^{-1}$ for $\left.r \in\right] 0,1$. Letting $r \rightarrow 1$ we get $\theta_{A_{0}}(x) \leq t<\bar{t}$. Hence our claim is true. For the last claim take $x^{*} \in A^{+} \backslash A^{-}$and assume that $\left\langle x_{0}, x^{*}\right\rangle=0$ for some $x_{0} \in A_{0}$. Take $x \in A$. Then there exists $s>0$ such that $x^{\prime}:=(1+s) x_{0}-s x \in A$. It follows that $0 \leq\left\langle x^{\prime}, x^{*}\right\rangle=-s\left\langle x, x^{*}\right\rangle \leq 0$, whence $\left\langle x, x^{*}\right\rangle=0$. Hence $-x^{*} \in A^{+}$, a contradiction.

Using Proposition 5 for $A$ replaced by $A_{0}$ we obtain

$$
\varsigma_{A}\left(x^{*}\right) \leq \inf \left\{\theta_{A}(x) \mid x \in \mathbb{P} A,\left\langle x, x^{*}\right\rangle=1\right\} \leq \inf \left\{\theta_{A}(x) \mid x \in \mathbb{P} A_{0},\left\langle x, x^{*}\right\rangle=1\right\}=\varsigma_{A_{0}}\left(x^{*}\right)
$$

for every $x^{*} \in A_{0}^{\#} \supset A^{+} \backslash A^{-}$. The conclusion follows.

The next result is a kind of converse of Proposition 6.

Proposition 7 Let $A \subset X \backslash\{0\}$ be a nonempty closed convex set such that $A=[1, \infty[A$, that is, $A$ is semi-conic. Then $A_{\infty}=\operatorname{cl}(\mathbb{P} A)$. Moreover, for every $x \in A_{\infty} \backslash\left(-A_{\infty}\right)$ one has

$$
\theta_{A}(x)=\sup \left\{\varsigma_{A}\left(x^{*}\right) \mid x^{*} \in A^{+},\left\langle x, x^{*}\right\rangle=1\right\} .
$$

Proof. We have that $x+s y=(1+s)\left(\frac{1}{1+s} x+\frac{s}{1+s} y\right) \in A$ for all $x, y \in A$ and $s \geq 0$ because $A$ is convex and $\left[1, \infty\left[A \subset A\right.\right.$. Hence $A \subset A_{\infty}$, and $\operatorname{socl}(\mathbb{P} A) \subset A_{\infty}$. On the other hand, for $u \in A_{\infty}$ we have that there exist the nets $\left(x_{i}\right)_{i \in I} \subset A$ and $\left(t_{i}\right)_{i \in I} \subset \mathbb{P}$ with $t_{i} \rightarrow 0$ and $t_{i} x_{i} \rightarrow u$. It follows that $u \in \operatorname{cl}(\mathbb{P} A)$. Hence $A_{\infty}=\operatorname{cl}(\mathbb{P} A)$.

First observe that for $s x \in A$ and $x^{*} \in X^{*}$ with $\left\langle x, x^{*}\right\rangle=1$ we have that $s=\left\langle s x, x^{*}\right\rangle \geq \varsigma_{A}\left(x^{*}\right)$. This proves the inequality $\geq$ in (16) for every $x \in X$. Take $x \in A_{\infty} \backslash\left(-A_{\infty}\right)$ and $0<s<\theta_{A}(x)$; this 
is possible because $\theta_{A}\left(x^{\prime}\right)>0$ for every $x^{\prime} \in X(A$ being closed and $0 \notin A)$. Then $s x \notin A$. Using a separation theorem we get $x^{*} \in X^{*}$ and $r \in \mathbb{R}$ such that

$$
\left\langle s x, x^{*}\right\rangle<r \leq\left\langle y, x^{*}\right\rangle \quad \forall y \in A .
$$

Since $A=A+A_{\infty}$ we obtain that $\left\langle u, x^{*}\right\rangle \geq 0$ for all $u \in A_{\infty}$, that is, $x^{*} \in\left(A_{\infty}\right)^{+}=(\mathbb{P} A)^{+}=A^{+}$. Because $x \in A_{\infty}$ we have that $\left\langle x, x^{*}\right\rangle \geq 0$, and so $r>0$. If $\left\langle x, x^{*}\right\rangle>0$ we may (and do) assume that $\left\langle x, x^{*}\right\rangle=1$. From (17) we obtain that $s<r \leq \varsigma_{A}\left(x^{*}\right)$. Assume now that $\left\langle x, x^{*}\right\rangle=0$. Because $x \in A_{\infty} \backslash\left(-A_{\infty}\right)$, there exists $\bar{x}^{*} \in A_{\infty}^{+}=A^{+}$such that $\left\langle x, \bar{x}^{*}\right\rangle=1$. Then $x_{t}^{*}:=t x^{*}+\bar{x}^{*} \in A^{+}$and $\left\langle x, x_{t}^{*}\right\rangle=1$ for $t \in \mathbb{R}_{+}$. Moreover, $\varsigma_{A}\left(x_{t}^{*}\right) \geq t \varsigma_{A}\left(x^{*}\right)+\varsigma_{A}\left(\bar{x}^{*}\right) \geq t r+\varsigma_{A}\left(\bar{x}^{*}\right)>s$ for $t>0$ sufficiently large. Hence (16) holds.

Of course, in the conditions of Proposition 7, if $-x \in A_{\infty}$ then $\left\langle x, x^{*}\right\rangle \leq 0$ for every $x^{*} \in A^{+}$, and so (16) does not hold (the term in the right hand side of (16) is $-\infty$ ).

Note that the supremum in (16) could be not attained. To see this consider

$$
A:=\left\{(x, y) \in \mathbb{R}^{2} \mid x \in[0,1], y \geq 1-\left(2 x-x^{2}\right)^{1 / 2}\right\} \cup\left(\left[1, \infty\left[\times \mathbb{R}_{+}\right) .\right.\right.
$$

Then $A_{\infty}=\mathbb{R}_{+}^{2}=A^{+}, \varsigma_{A}(a, 1)=1+a-\left(1+a^{2}\right)^{1 / 2}<1=\theta(0,1)$ for every $a \geq 0$.

Proposition 8 Let $\emptyset \neq A \subset X$. Then

$$
\begin{aligned}
\sigma_{A}\left(x^{*}\right) & =\sup \left\{\vartheta_{A}(x) \cdot\left\langle x, x^{*}\right\rangle \mid x \in\left[x^{*}>0\right] \cap \mathbb{P} A\right\} \\
& =\sup \left\{\vartheta_{A}(x) \cdot h\left\langle x, x^{*}\right\rangle \mid x \in\left[x^{*} \geq 0\right] \cap \mathbb{P} A\right\} \\
& =\sup \left\{\vartheta_{A}(x) \cdot h\left\langle x, x^{*}\right\rangle \mid x \in\left[x^{*} \geq 0\right] \cap A\right\} \\
& =\sup \left\{\vartheta_{A}(x) \cdot h\left\langle x, x^{*}\right\rangle \mid x \in\left[x^{*} \geq 0\right] \cap A^{++}\right\} \\
& =\sup \left\{\vartheta_{A}(x) \mid x \in \mathbb{P} A,\left\langle x, x^{*}\right\rangle=1\right\} \\
& =\sup \left\{\vartheta_{A}(x) \mid x \in X,\left\langle x, x^{*}\right\rangle=1\right\}>0
\end{aligned}
$$

for every $x^{*} \in X^{*} \backslash A^{-}$. Moreover, if $0 \in \overline{\operatorname{conv}} A$ then for every $x^{*} \in X^{*}$ one has

$$
\sigma_{A}\left(x^{*}\right)=0 \vee \sup \left\{\vartheta_{A}(x) \mid x \in X,\left\langle x, x^{*}\right\rangle=1\right\}
$$

Proof. Note first that $\sigma_{A}\left(x^{*}\right)>0$ if and only if $x^{*} \in X^{*} \backslash A^{-}$. Let $x^{*} \in X^{*} \backslash A^{-}$; then there exists $x \in A$ such that $\left\langle x, x^{*}\right\rangle>0$, and so $\left[x^{*}>0\right] \cap \mathbb{P} A \neq \emptyset$. Then

$$
\begin{aligned}
\sigma_{A}\left(x^{*}\right) & =\sup \left\{\left\langle x^{\prime}, x^{*}\right\rangle \mid x^{\prime} \in A\right\} \\
& =\sup \left\{t\left\langle x, x^{*}\right\rangle \mid t>0, x \in X, t x=x^{\prime} \in A\right\} \\
& =\sup \left\{t\left\langle x, x^{*}\right\rangle \mid t>0, x \in\left[x^{*}>0\right] \cap \mathbb{P} A, t x \in A\right\} \\
& =\sup _{x \in\left[x^{*}>0\right] \cap \mathbb{P} A}\left[\left\langle x, x^{*}\right\rangle \cdot \sup \{t>0 \mid t x \in A\}\right] \\
& =\sup \left\{\vartheta_{A}(x) \cdot\left\langle x, x^{*}\right\rangle \mid x \in\left[x^{*}>0\right] \cap \mathbb{P} A\right\}>0,
\end{aligned}
$$

that is, (18) holds. Because for $x \in\left[x^{*}=0\right] \cap \mathbb{P} A$ we have $\vartheta_{A}(x) \cdot_{h}\left\langle x, x^{*}\right\rangle=0$, the second equality holds, too. For the third equality one uses (2), while the fourth is a rewriting of the second one because for $x \in\left[x^{*} \geq 0\right] \cap A^{++} \backslash \mathbb{P} A$ we have that $\vartheta_{A}(x)=-\infty$ and $\left\langle x, x^{*}\right\rangle \geq 0$. Taking into account (2), from (18) we get immediately the fifth equality, while for the last equality observe that $\vartheta_{A}(x)=-\infty$ for $x \in X \backslash \mathbb{P} A$.

Assume that $0 \in \overline{\operatorname{conv}} A$; therefore, $\sigma_{A}\left(x^{*}\right)=\sigma_{\overline{\operatorname{conv}} A}\left(x^{*}\right) \geq 0$ for every $x^{*} \in X^{*}$. Set $\xi\left(x^{*}\right):=$ $\sup \left\{\vartheta_{A}(x) \mid x \in X,\left\langle x, x^{*}\right\rangle=1\right\}$ for $x^{*} \in X^{*}$. It is clear that $\xi(0)=-\infty$ and so (19) holds for $x^{*}=0$. If $x^{*} \in X^{*} \backslash A^{-}$(19) clearly follows from the first part. Let $0 \neq x^{*} \in A^{-}$. Then $\sigma_{A}\left(x^{*}\right)=0$ and $x \notin \mathbb{P} A$ for every $x \in\left[x^{*}=1\right]$. Therefore, $\xi\left(x^{*}\right)=-\infty$ and once again we have that (19) holds. 
Proposition 9 Let $A \subset X$ be such that $0 \in \overline{\operatorname{conv}} A$. Then for every $x \in X$ we have

$$
0 \vee \vartheta \overline{\operatorname{conv}} A(x)=\inf \left\{\sigma_{A}\left(x^{*}\right) \mid x^{*} \in X^{*},\left\langle x, x^{*}\right\rangle=1\right\} .
$$

Proof. Because $\sigma_{A}=\sigma_{\overline{\operatorname{conv}} A}$ we may (and do) assume that $A=\overline{\operatorname{conv}} A$. Set

$$
\eta(x):=\inf \left\{\sigma_{A}\left(x^{*}\right) \mid\left\langle x, x^{*}\right\rangle=1\right\}
$$

for $x \in X$. Using (19) it is clear that $0 \vee \vartheta_{A}(x) \leq \eta(x)$ for every $x \in X$. For the converse inequality we consider several cases.

a) $x=0$; then $\vartheta_{A}(x)=\infty$ and $\eta(x)=\infty$, and so (20) holds.

b) $x \in \mathbb{P} A \backslash\{0\}$ (hence $\vartheta_{A}(x)>0$ ). Take $s>\vartheta_{A}(x)$ (if possible). Then $s x \notin A$, and so, by a separation theorem, there exists $x^{*} \in X^{*}$ such that $\left\langle s x, x^{*}\right\rangle>\sigma_{A}\left(x^{*}\right) \geq 0$. Hence $\left\langle x, x^{*}\right\rangle>0$, and so we may (and do) assume that $\left\langle x, x^{*}\right\rangle=1$. Therefore, $\eta(x)<s$; the conclusion follows.

c) $x \notin \mathbb{P} A$. Consider the function $\varphi: X \rightarrow \overline{\mathbb{R}}$ defined by $\varphi(u)=t$ if $u=t x$ and $\varphi(u):=\infty$ else. Then $\varphi$ is a lsc convex function with $\varphi^{*}\left(x^{*}\right)=0$ if $\left\langle x, x^{*}\right\rangle=1$ and $\varphi^{*}\left(x^{*}\right)=\infty$ else. Moreover, we have that $\left(\iota_{A} \square \varphi\right)(u)=\inf \{t \in \mathbb{R} \mid u-t x \in A\}$. It is clear that $\left(\iota_{A} \square \varphi\right)(0)=0$. Let us prove that $\overline{\iota_{A} \square \varphi}(0)=0$. In the contrary case there exist $s>0$ and a net $\left(u_{i}\right)_{i \in I}$ converging to 0 such that $\left(\iota_{A} \square \varphi\right)\left(u_{i}\right)<-s$ for every $i \in I$. Therefore, for every $i \in I$ there exists $t_{i}>s$ with $x_{i}:=u_{i}+t_{i} x \in A$. We may (and do) assume that $t_{i} \rightarrow t \in[s, \infty]$. If $t<\infty$ then $t x \in \operatorname{cl} A=A$, a contradiction. If $t=\infty$ then $x=\lim t_{i}^{-1} x_{i}$, and so $x \in A_{\infty} \subset A$, again a contradiction. Therefore, $\overline{\iota_{A} \square \varphi}(0)=0$; in particular $\overline{\iota_{A} \square \varphi}$ is proper. Since $\left(\iota_{A} \square \varphi\right)^{*}=\sigma_{A}+\varphi^{*}$, we obtain (see [14]) that

$$
0=\overline{\iota_{A} \square \varphi}(0)=\left(\iota_{A} \square \varphi\right)^{* *}(0)=\left(\sigma_{A}+\varphi^{*}\right)^{*}(0)=-\inf _{x^{*} \in X^{*}}\left(\sigma_{A}+\varphi^{*}\right)\left(x^{*}\right)=-\eta(x) .
$$

Since $\vartheta_{A}(x)=-\infty$, we obtain that (20) holds in this case, too.

Observe that there exists $x \in X$ such that (20) is false if $0 \notin \overline{\operatorname{conv}} A$.

Note that using relations (1) and (3) one can establish duality results involving other combinations of the functions $\sigma_{A}, \varsigma_{A}, \mu_{A}, \nu_{A}, \theta_{A}$ and $\vartheta_{A}$.

\section{Duality relations involving $\sigma_{A}, \varsigma_{A}$ and $\psi_{A, k}$}

In [7, Rels. (18), (19)] the duality relations

$$
\varsigma_{A}\left(x^{*}\right)=\inf _{x}\left(\left\langle x, x^{*}\right\rangle-\psi_{A}(x) \cdot\left\langle k, x^{*}\right\rangle\right)
$$

and

$$
\psi_{A}(x)=\inf _{x^{*}} \frac{\left\langle x, x^{*}\right\rangle-\varsigma_{A}\left(x^{*}\right)}{\left\langle k, x^{*}\right\rangle}
$$

are given for $X=\mathbb{R}^{n}$ without mentioning from where $x$ and $x^{*}$ are taken. However in the context of [7] $A$ $(=L(y))$ is a convex set (included in $\left.\mathbb{R}_{+}^{n}\right)$ and $k\left(=g_{x}\right)$ is an element of $\mathbb{R}_{+}^{n} \backslash\{0\}$. In the sequel we shall try to find conditions which ensure the previous duality formulas.

In fact the first relation is true for $x^{*}$ with $\left\langle k, x^{*}\right\rangle>0$ taking $x \in X$, while in the second one must take $x^{*} \in X^{*}$ with $\left\langle k, x^{*}\right\rangle>0$ (or, equivalently, $\left\langle k, x^{*}\right\rangle=1$ ).

Proposition 10 Let $x^{*} \in X^{*}$ with $\left\langle k, x^{*}\right\rangle \geq 0$ and $\emptyset \neq A \subset B \subset X$. Then

$$
\varsigma_{A}\left(x^{*}\right)=\inf _{x \in B}\left(\left\langle x, x^{*}\right\rangle-\psi_{A, k}(x) \cdot{ }_{h}\left\langle k, x^{*}\right\rangle\right)
$$

and

$$
\sigma_{A}\left(x^{*}\right)=\sup _{x \in B}\left(\left\langle x, x^{*}\right\rangle+\psi_{A,-k}(x) \cdot \cdot_{e}\left\langle k, x^{*}\right\rangle\right) .
$$

If $\left\langle k, x^{*}\right\rangle>0$ then (23) and (24) hold even if $A=\emptyset$. 
Proof. Let first $\left\langle k, x^{*}\right\rangle>0$. If $A=\emptyset$ then (23) and (24) clearly hold. Assume that $A \neq \emptyset$. We have that

$$
\begin{aligned}
\inf _{x \in X}\left(\left\langle x, x^{*}\right\rangle-\psi_{A}(x) \cdot\left\langle k, x^{*}\right\rangle\right) & =\inf _{x \in X}\left(\left\langle x, x^{*}\right\rangle-\left\langle k, x^{*}\right\rangle \cdot \sup \{t \mid x-t k \in A\}\right) \\
& =\inf _{x \in X}\left(\inf \left\{\left\langle x, x^{*}\right\rangle-t\left\langle k, x^{*}\right\rangle \mid t \in \mathbb{R}, x-t k \in A\right\}\right) \\
& =\inf _{2}\left\{\left\langle x-t k, x^{*}\right\rangle \mid x \in X, t \in \mathbb{R}, x-t k \in A\right\} \\
& =\varsigma_{A}\left(x^{*}\right) .
\end{aligned}
$$

Hence, if $A \subset B \subset X$ then

$$
\begin{aligned}
\inf _{x \in X}\left(\left\langle x, x^{*}\right\rangle-\psi_{A}(x) \cdot\left\langle k, x^{*}\right\rangle\right) & \leq \inf _{x \in B}\left(\left\langle x, x^{*}\right\rangle-\psi_{A}(x) \cdot\left\langle k, x^{*}\right\rangle\right) \\
& \leq \inf _{x \in A}\left(\left\langle x, x^{*}\right\rangle-\psi_{A}(x) \cdot\left\langle k, x^{*}\right\rangle\right) \\
& \leq \inf _{x \in A}\left\langle x, x^{*}\right\rangle
\end{aligned}
$$

because $A \subset\left[\psi_{A} \geq 0\right]$. Therefore, (23) holds in this case.

Let now $\left\langle k, x^{*}\right\rangle=0$. Then

$$
\inf _{x \in X}\left(\left\langle x, x^{*}\right\rangle-\psi_{A}(x) \cdot{ }_{h}\left\langle k, x^{*}\right\rangle\right)=\inf \left\{\left\langle x, x^{*}\right\rangle \mid x \in \mathbb{R} k+A\right\}=\inf \left\{\left\langle x, x^{*}\right\rangle \mid x \in A\right\}=\varsigma_{A}\left(x^{*}\right),
$$

and so (23) holds in this case, too.

For obtaining (24) just use the formula $\sigma_{A}\left(x^{*}\right)=-\varsigma_{A}\left(-x^{*}\right)$ in (23).

If $\left\langle k, x^{*}\right\rangle<0$ relation (21) does not hold.

Example 1 Consider $A:=[1, \infty), k:=1$ and $x^{*}:=-1$, one has $\psi_{A}(x)=x-1$ for every $x \in X=\mathbb{R}$, $\varsigma_{A}\left(x^{*}\right)=-\infty$ and $\inf _{x \in X}\left\{\left\langle x, x^{*}\right\rangle-\psi_{A}(x) \cdot\left\langle k, x^{*}\right\rangle\right\}=-1$.

Corollary 1 Let $x^{*} \in X^{*}$ be such that $\left\langle k, x^{*}\right\rangle \geq 0$ and $A \subset X$. Then

$$
\varsigma_{A}\left(x^{*}\right)=\inf \left\{\left\langle x, x^{*}\right\rangle \mid x \in B\right\}
$$

provided $A \subset B \subset\left[\psi_{A, k} \geq 0\right]$ and

$$
\sigma_{A}\left(x^{*}\right)=\sup \left\{\left\langle x, x^{*}\right\rangle \mid x \in B\right\}
$$

provided $A \subset B \subset\left[\psi_{A,-k} \geq 0\right]$.

Proof. If $A=\emptyset$ then necessarily $B=\emptyset$, and so (25) and (26) hold. Assume that $A \neq \emptyset$. It is clear that $A \subset\left[\psi_{A} \geq 0\right]$; for $x \in\left[\psi_{A} \geq 0\right]$ clearly $\left\langle x, x^{*}\right\rangle-\psi_{A}(x){ }_{h}\left\langle k, x^{*}\right\rangle \leq\left\langle x, x^{*}\right\rangle$. Using the preceding proposition (for $B=X$ ) we get

$$
\begin{aligned}
\varsigma_{A}\left(x^{*}\right) & =\inf _{x \in X}\left(\left\langle x, x^{*}\right\rangle-\psi_{A}(x) \cdot{ }_{h}\left\langle k, x^{*}\right\rangle\right) \leq \inf _{x \in\left[\psi_{A} \geq 0\right]}\left(\left\langle x, x^{*}\right\rangle-\psi_{A}(x) \cdot{ }_{h}\left\langle k, x^{*}\right\rangle\right) \\
& \leq \inf _{x \in\left[\psi_{A} \geq 0\right]}\left\langle x, x^{*}\right\rangle \leq \inf _{x \in B}\left\langle x, x^{*}\right\rangle \leq \inf _{x \in A}\left\langle x, x^{*}\right\rangle=\varsigma_{A}\left(x^{*}\right) .
\end{aligned}
$$

Hence (25) holds. Replacing $x^{*}$ by $-x^{*}$ and $k$ by $-k$ in the preceding statement we get (26).

In what concerns relation (22) it is clear that one must have $\left\langle k, x^{*}\right\rangle \neq 0$. Also note that for $x \in X$ one has always

$$
\psi_{A}(x) \leq \inf \left\{\left\langle x, x^{*}\right\rangle-\varsigma_{A}\left(x^{*}\right) \mid x^{*} \in X^{*},\left\langle k, x^{*}\right\rangle=1\right\}=: \eta_{A}(x) .
$$

Indeed, let $x \in X, t \in \mathbb{R}$ and $x^{*} \in X^{*}$ be such that $x \in t k+A$ and $\left\langle k, x^{*}\right\rangle=1$. Then $\varsigma_{A}\left(x^{*}\right) \leq$ $\left\langle x-t k, x^{*}\right\rangle=\left\langle x, x^{*}\right\rangle-t$, that is, $t \leq\left\langle x, x^{*}\right\rangle-\varsigma_{A}\left(x^{*}\right)$. Therefore, the claim holds. 
However, one cannot expect equality for every set $A$. One reason is that $\psi_{A}$ is not necessarily concave, while $\eta_{A}$ is concave (and $\eta_{A}=\eta_{\overline{\operatorname{conv}} A}$ ); moreover, as seen in (7), $\psi_{A}=\psi_{A+\mathbb{R}_{+} k}$ which is not the case with $\varsigma_{A}$; however, $\varsigma_{A}\left(x^{*}\right)=\varsigma_{A+\mathbb{R}_{+} k}\left(x^{*}\right)$ if $\left\langle k, x^{*}\right\rangle>0$. It is natural to consider only $x^{*}$ with $\left\langle k, x^{*}\right\rangle>0$ (simple examples can be given with $A=A+\mathbb{R}_{+} k$ convex for which strict inequality holds in (27)).

It is known that for $A$ a nonempty closed convex set we have $\left(A_{\infty}\right)^{+}=\operatorname{cl}\left\{x^{*} \mid \varsigma_{A}\left(x^{*}\right)>-\infty\right\}$ (use f.i. [14, Exercise 2.23] for $\left.f=\iota_{A}\right)$. Since $\left(A_{\infty}\right)^{++}=A_{\infty}$, we have

$$
-k \in A_{\infty} \Longleftrightarrow\left[\varsigma_{A}\left(x^{*}\right)>-\infty \Rightarrow\left\langle k, x^{*}\right\rangle \leq 0\right],
$$

and so

$$
-k \notin A_{\infty} \Longleftrightarrow\left[\exists \bar{x}^{*} \in X^{*}: \varsigma_{A}\left(\bar{x}^{*}\right)>-\infty,\left\langle k, \bar{x}^{*}\right\rangle=1\right] .
$$

Note also that when $A$ is a nonempty closed (not necessarily convex) set and $k \in X$ is such that $-k \notin A_{\infty}$ we have that $A+\mathbb{R}_{+} k$ is closed and $\psi_{A}(x)<\infty$ for every $x \in X$.

Proposition 11 Assume that $A$ is a closed convex set. Then

$$
\psi_{A, k}(x)=\inf \left\{\left\langle x, x^{*}\right\rangle-\varsigma_{A}\left(x^{*}\right) \mid x^{*} \in X^{*},\left\langle k, x^{*}\right\rangle=1\right\} \quad \forall x \in X
$$

provided $-k \notin A_{\infty}$, and

$$
\psi_{A,-k}(x)=\inf \left\{\sigma_{A}\left(x^{*}\right)-\left\langle x, x^{*}\right\rangle \mid x^{*} \in X^{*},\left\langle k, x^{*}\right\rangle=1\right\} \quad \forall x \in X
$$

provided $k \notin A_{\infty}$.

Proof. If $A=\emptyset$ then clearly (29) and (30) hold.

Assume that $A \neq \emptyset$. As observed above $A+\mathbb{R}_{+} k$ is closed and $\psi_{A}(x)<\infty$ for every $x \in X$. Fix $x \in X$. By (27) we have that $\psi_{A}(x) \leq \eta_{A}(x)$. Take some $s \in \mathbb{R}$ such that $s>\psi_{A}(x)=\psi_{A+\mathbb{R}_{+}}(x)$; then $x-s k \notin A+\mathbb{R}_{+} k$. Because $A+\mathbb{R}_{+} k$ is convex and closed there exist $x_{0}^{*} \in X^{*}$ and $s_{0} \in \mathbb{R}$ such that $\left\langle x-s k, x_{0}^{*}\right\rangle\left\langle s_{0} \leq\left\langle a, x_{0}^{*}\right\rangle+t\left\langle k, x_{0}^{*}\right\rangle\right.$ for all $a \in A$ and $t \in \mathbb{R}_{+}$. It follows that $\left\langle k, x_{0}^{*}\right\rangle \geq 0$ and $\left\langle x, x_{0}^{*}\right\rangle-s\left\langle k, x_{0}^{*}\right\rangle\left\langle s_{0} \leq \varsigma_{A}\left(x_{0}^{*}\right)\right.$. If $\left\langle k, x_{0}^{*}\right\rangle \neq 0$, we may assume that $\left\langle k, x_{0}^{*}\right\rangle=1$ (replacing $x_{0}^{*}$ by $\left\langle k, x_{0}^{*}\right\rangle^{-1} x_{0}^{*}$ if necessary). Hence $\eta_{A}(x) \leq\left\langle x, x_{0}^{*}\right\rangle-\varsigma_{A}\left(x_{0}^{*}\right)<s\left\langle k, x_{0}^{*}\right\rangle=s$. Assume that $\left\langle k, x_{0}^{*}\right\rangle=0$. Because $-k \notin A_{\infty}$, by (28) there exists $\bar{x}^{*} \in X^{*}$ such that $\varsigma_{A}\left(\bar{x}^{*}\right)>-\infty$ and $\left\langle k, \bar{x}^{*}\right\rangle=1$. Then $\left\langle k, \bar{x}^{*}+t x_{0}^{*}\right\rangle=1$ and $\varsigma_{A}\left(\bar{x}^{*}+t x_{0}^{*}\right) \geq \varsigma_{A}\left(\bar{x}^{*}\right)+t \varsigma_{A}\left(x_{0}^{*}\right) \geq t s_{0}+\varsigma_{A}\left(\bar{x}^{*}\right)$ for $t \geq 0$, and so

$$
\begin{aligned}
\eta_{A}(x) & \leq \inf \left\{\left\langle x, \bar{x}^{*}+t x_{0}^{*}\right\rangle-\varsigma_{A}\left(\bar{x}^{*}+t x_{0}^{*}\right) \mid t \geq 0\right\} \\
& \leq\left\langle x, \bar{x}^{*}\right\rangle-\varsigma_{A}\left(\bar{x}^{*}\right)+\inf \left\{t \cdot\left[\left\langle x, x_{0}^{*}\right\rangle-s_{0}\right] \mid t \geq 0\right\} \\
& =-\infty
\end{aligned}
$$

Hence $\eta_{A}(x)<s$ in this case, too. It follows that $\eta_{A}(x) \leq \psi_{A}(x)$. Therefore, (29) holds. Replacing $k$ by $-k$ and $x^{*}$ by $-x^{*}$ in (29) we get (30).

\section{Other duality results}

Inspired by [7], we consider the nonempty set $T \subset X \times Y$, where $Y$ is another separated locally convex space with topological dual $Y^{*}$. As in [7] we associate the sets

$$
P(x):=\{y \in Y \mid(x, y) \in T\}, \quad L(y):=\{x \in X \mid(x, y) \in T\}
$$

for $x \in X$ and $y \in Y$; of course, $P(x)$ and $L(y)$ are convex (resp. closed) if $T$ is convex (resp. closed). Moreover, if $T$ is closed and convex and $u \in \operatorname{ker} T_{\infty}:=\left(T_{\infty}\right)^{-1}(0):=\left\{x \in X \mid(x, 0) \in T_{\infty}\right\}$, then $u \in(L(y))_{\infty}$ for any $y \in \operatorname{Pr}_{Y}(T)$; conversely, if $u \in(L(y))_{\infty}$ for some $y \in \operatorname{Pr}_{Y}(T)$ then $u \in \operatorname{ker} T_{\infty}$. A similar statement is valid with respect to the other variable. 
Define now the sets

$$
\begin{aligned}
& \bar{P}\left(x^{*}\right):=\left\{y \mid \exists x \in X: y \in P(x),\left\langle x, x^{*}\right\rangle \leq 1\right\}=\bigcup\left\{P(x) \mid\left\langle x, x^{*}\right\rangle \leq 1\right\} \subset \operatorname{Pr}_{Y}(T), \\
& \bar{L}\left(y^{*}\right):=\left\{x \mid \exists y \in Y: x \in L(y),\left\langle y, y^{*}\right\rangle \geq 1\right\}=\bigcup\left\{L(y) \mid\left\langle y, y^{*}\right\rangle \geq 1\right\} \subset \operatorname{Pr}_{X}(T),
\end{aligned}
$$

for $x^{*} \in X^{*}$ and $y^{*} \in Y^{*}$.

\section{Lemma 1 Let $T \subset X \times Y$ be nonempty.}

(a) If $T$ is convex then $\bar{P}\left(x^{*}\right)$ and $\bar{L}\left(y^{*}\right)$ are convex for all $x^{*} \in X^{*}$ and $y^{*} \in Y^{*}$.

(b) If $T$ is closed and $\operatorname{dim} X<\infty$ then $\bar{P}\left(x^{*}\right)$ is closed for every $x^{*} \in\left(\operatorname{ker} T_{\infty}\right)^{\#}$.

(c) If $T$ is closed and $\operatorname{dim} Y<\infty$ then $\bar{L}\left(y^{*}\right)$ is closed for every $y^{*} \in-\left(T_{\infty}(0)\right)^{\#}$.

Proof. (a) Let $y_{1}, y_{2} \in \bar{P}\left(x^{*}\right)$ and $s \in[0,1]$. There exist $x_{1}, x_{2} \in X$ with $y_{1} \in P\left(x_{1}\right)$ and $y_{2} \in P\left(x_{2}\right)$, that is, $\left(x_{1}, y_{1}\right),\left(x_{2}, y_{2}\right) \in T$ and $\left\langle x_{1}, x^{*}\right\rangle \leq 1,\left\langle x_{2}, x^{*}\right\rangle \leq 1$. Hence $\left\langle s x_{1}+(1-s) x_{2}, x^{*}\right\rangle \leq 1$ and, by the convexity of $T,\left(s x_{1}+(1-s) x_{2}, s y_{1}+(1-s) y_{2}\right) \in T$, that is, $s y_{1}+(1-s) y_{2} \in P\left(s x_{1}+(1-s) x_{2}\right)$. Hence $s y_{1}+(1-s) y_{2} \in \bar{P}\left(x^{*}\right)$. The convexity of $\bar{L}\left(y^{*}\right)$ follows similarly.

(b) Because $\operatorname{dim} X<\infty$ we may (and do) assume $X$ is a normed space. Fix some $x^{*} \in\left(\operatorname{ker} T_{\infty}\right)^{\#}$ and consider $y \in \operatorname{cl}\left(\bar{P}\left(x^{*}\right)\right)$, that is, there exists $\left(y_{i}\right)_{i \in I} \subset \bar{P}\left(x^{*}\right)$ with $y_{i} \rightarrow y$. For every $i \in Y$ there exists $x_{i} \in X$ with $\left(x_{i}, y_{i}\right) \in T$ and $\left\langle x_{i}, x^{*}\right\rangle \leq 1$. Assume that $t_{i}:=\left\|x_{i}\right\| \rightarrow \infty$ (on a subnet); hence $t_{i} \in \mathbb{P}$ for $i \geq i_{0}$. Passing to a subnet if necessary, $t_{i}^{-1} x_{i} \rightarrow u \neq 0$. Since $t_{i}^{-1} \rightarrow 0$ and $t_{i}^{-1}\left(x_{i}, y_{i}\right) \rightarrow(u, 0)$, we have that $u \in \operatorname{ker} T_{\infty}$. Moreover, clearly, $t_{i}^{-1} \geq\left\langle t_{i}^{-1} x_{i}, x^{*}\right\rangle \rightarrow\left\langle u, x^{*}\right\rangle$, and so $\left\langle u, x^{*}\right\rangle \leq 0$, contradicting the fact that $x^{*} \in\left(\operatorname{ker} T_{\infty}\right)^{\#}$. Therefore, there exists some $i_{0}$ such that $\left(x_{i}\right)_{i \geq i_{0}}$ is bounded, and so we may (and do) assume that $x_{i} \rightarrow x \in X$; hence $\left\langle x, x^{*}\right\rangle \leq 1$. Because $T$ is closed we obtain that $(x, y) \in T$, and so $y \in P(x) \subset \bar{P}\left(x^{*}\right)$.

(c) The proof is similar to that of (b).

Throughout this section $k \in X \backslash\{0\}$ and $l \in Y \backslash\{0\}$ are fixed elements. Using Proposition 3 we obtain that

$$
\psi_{\bar{L}\left(y^{*}\right), k}=\sup \left\{\psi_{L(y), k} \mid\left\langle y, y^{*}\right\rangle \geq 1\right\}, \quad \psi_{\bar{P}\left(x^{*}\right), l}=\sup \left\{\psi_{P(x), l} \mid\left\langle x, x^{*}\right\rangle \leq 1\right\} .
$$

Proposition 12 Assume that $T$ is a nonempty closed convex set such that $0 \in P(x)$ for every $x \in \operatorname{Pr}_{X}(T)$. Then

$$
L(y)=\bigcap\left\{\bar{L}\left(y^{*}\right) \mid\left\langle y, y^{*}\right\rangle \geq 1\right\} \quad \forall y \in Y .
$$

Moreover, if $k \in \operatorname{ker} T_{\infty}$ then

$$
\psi_{L(y), k}=\inf \left\{\psi_{\bar{L}\left(y^{*}\right), k} \mid\left\langle y, y^{*}\right\rangle \geq 1\right\} \quad \forall y \in Y
$$

Proof. Let us set $\widetilde{L}(y):=\bigcap\left\{\bar{L}\left(y^{*}\right) \mid\left\langle y, y^{*}\right\rangle \geq 1\right\}$ for $y \in Y$. From (32) it is clear that $L(y) \subset \widetilde{L}(y)$ for every $y \in Y$. Fix $\bar{y} \in Y$ and let us show that $\widetilde{L}(\bar{y}) \subset L(\bar{y})$. Take some $\bar{x} \in X \backslash L(\bar{y})$. If $\bar{x} \notin \operatorname{Pr}_{X}(T)$, by (32) we have that $\bar{x} \notin \widetilde{L}(\bar{y})$. So let $\bar{x} \in \operatorname{Pr}_{X}(T)$; hence $(\bar{x}, 0) \in T$. Because $(\bar{x}, \bar{y}) \notin T$, by a separation theorem, there exist $\left(\bar{x}^{*}, \bar{y}^{*}\right) \in X^{*} \times Y^{*}$ and $s \in \mathbb{R}$ such that

$$
\left.\left\langle\bar{x}, \bar{x}^{*}\right\rangle+\left\langle\bar{y}, \bar{y}^{*}\right\rangle>s\right\rangle\left\langle x, \bar{x}^{*}\right\rangle+\left\langle y, \bar{y}^{*}\right\rangle \quad \forall(x, y) \in T .
$$

Since $(\bar{x}, 0) \in T$ we obtain that $\left\langle\bar{y}, \bar{y}^{*}\right\rangle>s_{0}:=s-\left\langle\bar{x}, \bar{x}^{*}\right\rangle>0$. We may (and do) assume that $s_{0}=1$. It follows that $\left\langle\bar{y}, \bar{y}^{*}\right\rangle>1$ and $(\bar{x}, y) \in T \Rightarrow\left\langle y, \bar{y}^{*}\right\rangle<1$. The last implication shows that $\bar{x} \notin L(y)$ for $\left\langle y, \bar{y}^{*}\right\rangle \geq 1$, that is, $\bar{x} \notin \bar{L}\left(\bar{y}^{*}\right)$. Since $\left\langle\bar{y}, \bar{y}^{*}\right\rangle \geq 1$, we have that $\bar{x} \notin \widetilde{L}(\bar{y})$.

Assume that $k \in \operatorname{ker} T_{\infty}$. Because $(k, 0) \in T_{\infty}$ we have that $L(y)+\mathbb{R}_{+} k=L(y)$ for every $y \in Y$, and so $\bar{L}\left(y^{*}\right)+\mathbb{R}_{+} k=\bar{L}\left(y^{*}\right)$ for every $y^{*} \in Y^{*}$. Applying Propositions 12 and 3 (with $K=X$ ) we get the conclusion. The proof is complete. 
Proposition 13 Assume that $T \subset X \times Y$ is a nonempty closed convex set. Then

$$
P(x)=\bigcap\left\{\bar{P}\left(x^{*}\right) \mid\left\langle x, x^{*}\right\rangle \leq 1\right\} \quad \forall x \in \operatorname{ker} T_{\infty}
$$

Moreover, assume that the closed set $F \subset Y$ and $l \in Y \backslash\{0\}$ are such that

$$
P_{Y}(T) \subset F \quad \text { and } \quad[(x, y) \in T, t \geq 0, y+t l \in F] \Longrightarrow(x, y+t l) \in T .
$$

Then

$$
\psi_{P(x), l}=\inf \left\{\psi_{\bar{P}\left(x^{*}\right), l} \mid\left\langle x, x^{*}\right\rangle \leq 1\right\} \quad \forall x \in \operatorname{ker} T_{\infty}
$$

PROOF. Let us set

$$
\widetilde{P}(x):=\bigcap\left\{\bar{P}\left(x^{*}\right) \mid\left\langle x, x^{*}\right\rangle \leq 1\right\}, \quad x \in X
$$

From (31) it is clear that $P(x) \subset \widetilde{P}(x)$ for every $x \in X$. Fix $\bar{x} \in \operatorname{ker} T_{\infty}$ and let us show $\widetilde{P}(\bar{x}) \subset P(\bar{x})$. Take some $\bar{y} \in Y \backslash P(\bar{x})$. Because $(\bar{x}, \bar{y}) \notin T$, by a separation theorem, there exist $\left(\bar{x}^{*}, \bar{y}^{*}\right) \in X^{*} \times Y^{*}$ and $s \in \mathbb{R}$ such that

$$
\left\langle\bar{x}, \bar{x}^{*}\right\rangle+\left\langle\bar{y}, \bar{y}^{*}\right\rangle<s<\left\langle x, \bar{x}^{*}\right\rangle+\left\langle y, \bar{y}^{*}\right\rangle \quad \forall(x, y) \in T
$$

Fixing some $\left(x_{0}, y_{0}\right) \in T$ we have that $\left(x_{0}+u, y_{0}\right) \in T$ for every $u \in \operatorname{ker} T_{\infty}$. From (40) we obtain that $\left\langle u, \bar{x}^{*}\right\rangle \geq 0$ for every $u \in \operatorname{ker} T_{\infty}$. In particular $\left\langle\bar{x}, \bar{x}^{*}\right\rangle \geq 0$. Using again (40) we obtain that $\left\langle\bar{y}, \bar{y}^{*}\right\rangle \leq\left\langle\bar{x}, \bar{x}^{*}\right\rangle+\left\langle\bar{y}, \bar{y}^{*}\right\rangle\left\langle s\right.$, whence $s_{0}:=s-\left\langle\bar{y}, \bar{y}^{*}\right\rangle>0$. We may (and do) assume that $s_{0}=1$. It follows that $\left\langle\bar{x}, \bar{x}^{*}\right\rangle<1$ and $(x, \bar{y}) \in T \Rightarrow\left\langle x, \bar{x}^{*}\right\rangle>1$. The last implication shows that $\bar{y} \notin P(x)$ for $\left\langle x, \bar{x}^{*}\right\rangle \leq 1$, that is, $\bar{y} \notin \bar{P}\left(\bar{x}^{*}\right)$. Since $\left\langle\bar{x}, \bar{x}^{*}\right\rangle \leq 1$, we have that $\bar{y} \notin \widetilde{P}(\bar{x})$.

Assume that $F$ and $l$ satisfy (37). Condition (37) shows that $\left(P(x)+\mathbb{R}_{+} l\right) \cap F=P(x)$ for every $x \in X$. By Proposition 2 we obtain that $\left(\bar{P}\left(x^{*}\right)+\mathbb{R}_{+} l\right) \cap F=\bar{P}\left(x^{*}\right)$ for every $x^{*} \in X^{*}$. Using Proposition 3 we obtain that $\inf \left\{\psi_{\bar{P}\left(x^{*}\right), l} \mid\left\langle x, x^{*}\right\rangle \leq 1\right\}=\psi_{\widetilde{P}(x), l}$. Since $P(x)=\widetilde{P}(x)$ we get (38).

The next example shows that for $x \in X \backslash \operatorname{ker} T_{\infty}$ it is possible that (36) is not verified under the hypotheses of Proposition 13.

Example 2 Let $X=Y=\mathbb{R}, K:=\mathbb{R}_{+}, a \in \mathbb{R}$ and $T_{a}:=\left\{(x, y) \in X \times Y \mid y \geq 0, x \geq a+y^{2}\right\}$. Then $P_{a}(x)=[0, \sqrt{x-a}]$ for $x \geq a$ and $P_{a}(x)=\emptyset$ for $x<a$. Moreover, for $a \leq 0$ one has

$$
\bar{P}_{a}(u)=\left\{\begin{array}{ll}
{[0, \infty)} & \text { if } u \leq 0, \\
{[0, \sqrt{1 / u-a}]} & \text { if } u>0,
\end{array} \quad \widetilde{P}_{a}(x)= \begin{cases}{[0, \sqrt{-a}]} & \text { if } x \leq 0 \\
{[0, \sqrt{x-a}]} & \text { if } x>0\end{cases}\right.
$$

while for $a>0$ one has

$$
\bar{P}_{a}(u)=\left\{\begin{array}{ll}
{[0, \infty)} & \text { if } u \leq 0, \\
{[0, \sqrt{1 / u-a}]} & \text { if } 0<u \leq 1 / a, \\
\emptyset & \text { if } u>1 / a,
\end{array} \quad \widetilde{P}_{a}(x)= \begin{cases}\emptyset & \text { if } x<a \\
{[0, \sqrt{x-a}]} & \text { if } x \geq a\end{cases}\right.
$$

Hence $\widetilde{P}_{a}(x)=P_{a}(x)$ for every $x \in K$ (and every $\left.a\right)$, but $\widetilde{P}_{a}(x) \neq P_{a}(x)$ for $x \in X \backslash K$ when $a \leq 0$ ( $\widetilde{P}$ being defined in (39)).

Note that relations (33), (35) and (38), as well as (34) and (36) can be interpreted also as duality results. 


\section{Connections with duality results in economics literature}

Taking $A:=\{x \in \mathcal{X} \mid u(x) \geq u\}$ and $B:=\mathcal{X}$ Proposition 10 extends [11, Prop. 2.4] because $b(x, u)=$ $\psi_{A, g}(x)$. It also extends [11, Prop. 4.1]. Indeed, using the notation from [11], we have $\pi(p)=\sigma_{\mathcal{Y}}(p)$ and $\sigma(g ; y)=\varphi_{-\mathcal{Y}, g}(y)$. So, from (24) and (9) we get for $g \cdot p>0, A:=-\mathcal{Y}, k:=g$ and $x^{*}:=p$,

$$
\pi(p)=\sigma_{\mathcal{Y}}(p)=\sup _{x \in \mathbb{R}^{m}}\left(\langle x, p\rangle+\psi_{\mathcal{Y},-g}(x) \cdot\langle g, p\rangle\right)=\sup _{x \in \mathbb{R}^{m}}(x \cdot p-\sigma(g ; y) g \cdot p),
$$

that is, the conclusion of [11, Prop. 4.1].

In several papers on production analysis a technology is a nonempty set $T \subset \mathbb{R}_{+}^{n} \times \mathbb{R}_{+}^{m}$ with $n, m \geq 1$ satisfying several axioms among the next ones (see [4, p. 353]):

(A1) $T$ is closed.

(A2) Inputs and outputs are freely disposable; i.e., $(x, y) \in T,\left(x^{\prime}, y^{\prime}\right) \in \mathbb{R}^{n} \times \mathbb{R}^{m}$ and $x^{\prime} \geq x, 0 \leq y^{\prime} \leq y$ imply $\left(x^{\prime}, y^{\prime}\right) \in T$ (here $x^{\prime} \geq x$ means $x^{\prime}-x \in \mathbb{R}_{+}^{n}$ and $y^{\prime} \leq y$ means $y-y^{\prime} \in \mathbb{R}_{+}^{m}$ ).

(A3) There is no free lunch; i.e., $(0, y) \in T$ implies $y=0$.

(A4) Doing nothing is feasible; i.e., $(0,0) \in T$.

(A5) $T$ is convex.

Sometimes instead of the (free) disposability axiom (A2) one uses the weak disposability axiom

(A2') $(x, y) \in T, s \in[1, \infty[$ and $t \in[0,1]$ imply $(s x, y) \in T$ and $(x, t y) \in T$.

Note that axiom (A2) is written in the form: "if $(x, y) \in T$ and $\left(x^{\prime},-y^{\prime}\right) \geq(x,-y)$, then $\left(x^{\prime}, y^{\prime}\right) \in T$ " in [4] (and other articles); this is equivalent to (A2) if one asks $\left(x^{\prime}, y^{\prime}\right) \in \mathbb{R}_{+}^{n} \times \mathbb{R}_{+}^{m}$. Because the technology $T$ is perfectly determined by the multifunction $P: \mathbb{R}_{+}^{n} \rightrightarrows \mathbb{R}_{+}^{m}$ or $L: \mathbb{R}_{+}^{m} \rightrightarrows \mathbb{R}_{+}^{n}$, sometimes one mentions the axioms in terms of $P$ or $L$. We set $X:=\mathbb{R}^{n}$ and $Y:=\mathbb{R}^{m}$. Note that when (A1) and (A2') hold we have that $P(x)$ is a nonempty closed set with $[0,1] P(x)=P(x)$ for every $x \in \operatorname{Pr}_{X}(T)$ and $L(y)$ is a nonempty closed set with $\left[1, \infty\left[L(y)=L(y)\right.\right.$ for every $y \in \operatorname{Pr}_{Y}(T)$. Taking into account (4) and (5) the preceding remark proves that for $(x, y) \in X \times Y$ one has the equivalences

$$
D_{i}(y, x) \geq 1 \Longleftrightarrow x \in L(y) \Longleftrightarrow(x, y) \in T \Longleftrightarrow y \in P(x) \Longleftrightarrow D_{o}(x, y) \leq 1
$$

mentioned in [4, p. 353], where $D_{i}(y, x):=\nu_{L(y)}(x)$ and $D_{o}(x, y):=\mu_{P(x)}(y) ; D_{i}$ and $D_{o}$ are the Shephard input and output distance functions. Besides $D_{i}$ and $D_{o}$ in production analysis other functions are considered, too (see [7] and the references therein):

$$
\begin{gathered}
\vec{D}_{T}\left(x, y ;-g_{x}, g_{y}\right):=\psi_{T,\left(g_{x},-g_{y}\right)}(x, y), \\
\vec{D}_{o}\left(x, y ; g_{y}\right):=\psi_{P(x),-g_{y}}(y), \quad \vec{D}_{i}\left(x, y ;-g_{x}\right):=\psi_{L(y), g_{x}}(x), \\
\overrightarrow{I D}_{o}\left(w^{\prime}, y ; g_{y}\right):=\psi_{\bar{P}\left(w^{\prime}\right),-g_{y}}(y), \quad \overrightarrow{I D}_{i}\left(x, p^{\prime} ;-g_{x}\right):=\psi_{\bar{L}\left(p^{\prime}\right), g_{x}}(x),
\end{gathered}
$$

where $\bar{P}\left(w^{\prime}\right)$ and $\bar{L}\left(p^{\prime}\right)$ are defined as in (31), (32) for $w^{\prime} \in X^{*}=\mathbb{R}^{n}$ and $p^{\prime} \in Y^{*}=\mathbb{R}^{m}$ (corresponding to the sets $I P(w / C)$ and $I L(p / R))$; here $g_{x} \in \mathbb{R}_{+}^{n}$ and $g_{y} \in \mathbb{R}_{+}^{m}$ are nonnull in (42), (43) and $\left(g_{x}, g_{y}\right) \neq(0,0)$ in (41). Moreover,

$$
\begin{aligned}
\Pi(p, w):= & \sigma_{T}(-w, p), \quad R(x, p):=\sigma_{P(x)}(p), \quad C(y, w):=\varsigma_{L(y)}(w), \\
& \operatorname{IR}\left(w^{\prime}, p\right):=\sigma_{\bar{P}\left(w^{\prime}\right)}(p), \quad I C\left(p^{\prime}, w\right):=\varsigma_{\bar{L}\left(p^{\prime}\right)}(w) .
\end{aligned}
$$


Assuming that the axioms (A1), (A2'), (A3), (A4), (A5) hold and using also (3), from Propositions 8, 9, 6 and 7 we obtain that

$$
\begin{array}{ll}
R(x, p)=\sup _{y}\left\{\frac{p y}{D_{o}(x, y)} \mid p y>0\right\} & \forall x \in \operatorname{Pr}_{X}(T), \quad \forall p \in \mathbb{R}^{m} \backslash\{0\}, \\
\frac{1}{D_{o}(x, y)}=\inf _{p}\left\{\frac{R(x, p)}{p y} \mid p y>0\right\} & \forall x \in \operatorname{Pr}_{X}(T), \quad \forall y \in \mathbb{R}^{m} \backslash\{0\}, \\
C(y, w)=\inf _{x}\left\{\frac{w x}{D_{i}(y, x)} \mid w x>0\right\} & \forall y \in \operatorname{Pr}_{Y}(T), \quad \forall w \in \mathbb{R}_{+}^{n} \backslash\{0\}, \\
\frac{1}{D_{i}(y, x)}=\sup _{w}\left\{\frac{C(y, w)}{w x} \mid w x>0\right\} & \forall y \in \operatorname{Pr}_{Y}(T), \quad \forall x \in \mathbb{R}_{+}^{n} \backslash\{0\},
\end{array}
$$

respectively; in fact an attentive analysis shows that some hypotheses can be weakened. Such duality results are mentioned in [6, Rels. (10), (11)]; here one says "Shephard (Refs. 1, 9) proved that $C(y, w)$ is dual to $D_{i}(y, x)$ and that $R(x, p)$ is dual to $D_{o}(x, y)$. His duality theorems were stated as constrained optimization problems. Here, we follow Färe and Primont (Ref. 3) and state the dualities as unconstrained optimization problems".

In the sequel we assume that $T$ satisfies the axioms (A1)-(A5). In this situation $T_{\infty}$ is a subset of $\mathbb{R}_{+}^{n} \times \mathbb{R}_{+}^{m}$ and satisfies the axioms (A1)-(A5), too; in particular $\mathbb{R}_{+}^{n} \times\{0\} \subset T_{\infty}$, ker $T_{\infty}=\mathbb{R}_{+}^{n}$ and $T_{\infty}(0)=\{0\}$. Moreover, $\operatorname{Pr}_{X}(T)=\mathbb{R}_{+}^{n}$ and $P(x)$ is a compact convex set containing 0 for every $x \in \operatorname{Pr}_{X}(T)$, and $L(y)$ is a nonempty closed convex set with $(L(y))_{\infty}=\mathbb{R}_{+}^{m}$ for every $y \in \operatorname{Pr}_{Y}(T)$. From axiom (A2) we obtain that

$$
T=\left(T+\mathbb{R}_{+} k\right) \cap\left(\mathbb{R}_{+}^{n} \times \mathbb{R}_{+}^{m}\right) \quad \forall k \in \mathbb{R}_{+}^{n} \times\left(-\mathbb{R}_{+}^{m}\right)
$$

hence (10) holds for $k \in \mathbb{R}_{+}^{n} \times\left(-\mathbb{R}_{+}^{m}\right)$ (and $\left.K:=\mathbb{R}_{+}^{n} \times \mathbb{R}_{+}^{m}\right)$. Moreover

$$
P(x)=\left(P(x)-\mathbb{R}_{+}^{m}\right) \cap \mathbb{R}_{+}^{m} \quad \forall x \in X \quad \text { and } \quad L(y)=\left(L(y)+\mathbb{R}_{+}^{n}\right) \cap \mathbb{R}_{+}^{n} \quad \forall y \in Y .
$$

Because

$$
-\left(g_{x},-g_{y}\right) \notin T_{\infty}\left(\subset \mathbb{R}_{+}^{n} \times \mathbb{R}_{+}^{m}\right), \quad-g_{x} \notin(L(y))_{\infty}\left(\subset \mathbb{R}_{+}^{n}\right) \quad \text { and } \quad g_{y} \notin(P(x))_{\infty}(=\{0\}),
$$

the functions $\vec{D}_{T}, \vec{D}_{o}, \vec{D}_{i}$ do not take the value $+\infty$; this is also true for $\vec{D}_{i}$ (because $-g_{x} \notin\left(\bar{L}\left(p^{\prime}\right)\right)_{\infty}$ $\left(\subset \mathbb{R}_{+}^{n}\right)$ and for $\overrightarrow{I D}_{o}$ when $w^{\prime} \in \mathbb{R}_{++}^{n}:=\operatorname{int} \mathbb{R}_{+}^{n}$, because $\bar{P}\left(w^{\prime}\right)$ is compact in this case. Indeed, by Lemma $1 \bar{P}\left(w^{\prime}\right)$ is closed. Assume that $\bar{P}\left(w^{\prime}\right)$ is not bounded. This means that there exists a sequence $\left(\left(x_{n}, y_{n}\right)\right)_{n \geq 1} \subset T$ with $\left\|y_{n}\right\| \rightarrow \infty$ and $\left\langle x_{n}, w^{\prime}\right\rangle \leq 1$ for every $n \geq 1$. We may assume that $\left\|\left(x_{n}, y_{n}\right)\right\|^{-1}\left(x_{n}, y_{n}\right) \rightarrow(u, v) \in T_{\infty} \subset \mathbb{R}_{+}^{n} \times \mathbb{R}_{+}^{m}$. Because $(0,0) \in T$, we get $(s u, s v) \in T$ for $s \in \mathbb{R}_{+}$. If $u=0$, then $(0, v) \in T$ and so $v=0$ by (A3), contradicting $\|(u, v)\|=1$. Hence $u \neq 0$. It follows that $\left\langle u, w^{\prime}\right\rangle \leq 0$, contradicting the fact that $u \in \mathbb{R}_{+}^{n} \backslash\{0\}$ and $w^{\prime} \in \mathbb{R}_{++}^{n}$. Hence $\bar{P}\left(w^{\prime}\right)$ is bounded.

Note that for $w \notin \mathbb{R}_{+}^{n}$, taking into account (A2), we have that $\Pi(p, w)=\infty, C(y, w)=-\infty$ (if $L(y) \neq \emptyset), I C\left(p^{\prime}, w\right)=-\infty$ (if $\bar{L}\left(p^{\prime}\right) \neq \emptyset$ ); hence, in those results involving these quantities we may take $w \in \mathbb{R}_{+}^{n}$. Using Proposition 1 we obtain that

$$
(x, y) \in T \Longleftrightarrow\left[(x, y) \in \mathbb{R}_{+}^{n} \times \mathbb{R}_{+}^{m} \text { and } \vec{D}_{T}\left(x, y ;-g_{x}, g_{y}\right) \geq 0\right]
$$

and

$$
y \in P(x) \Longleftrightarrow\left[y \in \mathbb{R}_{+}^{m} \text { and } \vec{D}_{o}\left(x, y ; g_{y}\right) \geq 0\right] ;
$$

taking into account (8) we see that $[7,(2)]$ and $[7,(5)])$ do not hold. 
Applying Proposition 10 we get [7, (16)] (and [4, (16)]; this is obtained under a differentiability assumption on solutions) for (those pairs $(p, w)$ with) $p g_{y}+w g_{x}>0,\left[7,(18)\right.$, (33)] for $w g_{x}>0$, and [7, (20), (35)] for $p g_{y}>0$. On the other hand, taking into account (44) and using Proposition 11 we get [7, (17), (19), (21)] in which the infimum is taken with respect to (w.r.t.) those $p$, w with $p g_{y}+w g_{x}>0$ (or equivalently $p g_{y}+w g_{x}=1$ ), w.r.t. $w$ with $w g_{x}>0$ and w.r.t. $p$ with $p g_{y}>0$, respectively. Moreover, by Lemma 1 we have that $\bar{P}\left(w^{\prime}\right)$ is a closed convex set containing 0 for every $w^{\prime} \in \mathbb{R}_{++}^{n}=\left(\operatorname{ker} T_{\infty}\right)^{\#}$ and $\bar{L}\left(p^{\prime}\right)$ is a closed convex set for every $p^{\prime} \in Y^{*}=\mathbb{R}^{m}=-\left(T_{\infty}(0)\right)^{\#}$. Using again Proposition 11 we get [7, (34)] in which the supremum is taken w.r.t. $w$ with $w g_{x}>0$ for every $p^{\prime} \in \mathbb{R}^{m}$ and [7, (36)] in which the infimum is taken w.r.t. $p$ with $p g_{y}>0$ for every $w^{\prime} \in \mathbb{R}_{++}^{n}$

We have that [7, (26)] and [7, (31)] follow immediately from (33) (if our interpretation, used throughout this section, that $C$ and $R$ are positive real numbers, is correct). Moreover, using Proposition 13 for $F=\mathbb{R}_{+}^{m}$ and $l=-g_{y}$ we get $[7,(27)]$ for every $x \in \operatorname{ker} T_{\infty}=\mathbb{R}_{+}^{n}$, while using Proposition 12 for $k=g_{x}$ we get [7, (32)] for every $y \in Y$.

Recently one considered technologies in which the output space $Y$ is a functions space. For example in [3] one considers the technology $\widetilde{T} \subset \mathbb{R}_{+}^{n} \times L^{2}\left(\Omega, \mathbb{P}, \mathbb{R}_{+}^{m}\right)$ where $\mathbb{P}$ is a probability measure. Note that the duality results established in [3] can be deduced from the duality results from Section 4.

Acknowledgement. I wish to thank Professors P. Jiménez Guerra and M. J. Muñoz-Bouzo for helpful discussions on the subject during my visit at U.N.E.D. in November 2007, as well as to thank Dr. R. I. Botg for several remarks and mainly for pointing out that similar arguments to those in part (c) of the proof of Proposition 9 can be used for the proof of Proposition 11. I was supported by the CNCSIS grant ID-PCE-379.

\section{References}

[1] Agrell, P. J., Bogetoft, P., Brock, M. And Tind, J., (2005). Efficiency evaluation with convex pairs, Adv. Model. Optim., 7, 211-237.

[2] Artzner, P., Delbaen, F., Eber, J.-M. And Heath, D., (1999). Coherent measures of risk, Math. Finance, 9, 203-228.

[3] Briec, W. ANd Cavaignac, L., (2009). An extension of the multi-output state-contingent production model, Econom. Theory, 39, 43-64.

[4] Chambers, R. G., Chung, Y. And Färe, R., (1998). Profit, directional distance functions, and Nerlovian efficiency, J. Optim. Theory Appl., 98, 351-364.

[5] Cherchye, L., Kuosmanen, T. And Post, T., (2000). Why convexify? An assessment of convexity axioms in DEA, working paper, Helsinki School of Economics.

[6] FÄre, R. And Grosskopf, S., (2000). On separability of the profit function, J. Optim. Theory Appl., 105, $609-620$

[7] Färe, R. And Primont, D., (2006). Directional duality theory, Econom. Theory, 29, 239-247.

[8] Gerstewitz (Tammer), Chr. And Iwanow, E., (1985). Dualität für nichtkonvexe Vektoroptimierungsprobleme, Wissenschaftliche Zeitschrift der Technischen Hochschule Ilmenau, 31, 2, 61-81.

[9] Göpfert, A., TAMmer, C., Riahi, H. And ZăLinesCU, C., (2003). Variational methods in partially ordered spaces, CMS Books in Mathematics/Ouvrages de Mathématiques de la SMC 17, Springer, New York.

[10] HAMEL, A. H., (2007). Translative sets and functions and its applications to risk measure theory and nonlinear separation, Preprint, University Halle-Wittenberg.

[11] Luenberger, D. G., (1992). New optimality principles for economic efficiency and equilibrium, J. Optim. Theory Appl., 75, 221-264. 
C. Zălinescu

[12] Penot, J.-P. And Zălinescu, C., (2000). Harmonic sum and duality, J. Convex Anal., 7 , 95-114.

[13] TAmmer, C. And ZăLinescu, C., Lipschitz properties of the scalarization function and applications, Optimization, DOI: 10.1080/02331930801951033.

[14] ZăLinESCU, C., (2002). Convex Analysis in General Vector Spaces, World Scientific, Singapore.

\section{Zălinescu}

University "Al. I. Cuza” Iaşi,

Faculty of Mathematics,

700506-Iaşi, Romania,

and Institute of Mathematics Octav Mayer, Iaşi, Romania, zalinesc@uaic.ro 